\title{
Radiological features of perivascular epithelioid cell tumours (PEComas) in a paediatric patient - Case report
}

\author{
Klaudia Karska ${ }^{1, A-F}{ }^{\oplus}$, Ilona Kozioł ${ }^{1, A-D \oplus}$, Magdalena Leśniewska ${ }^{1, A-D \oplus}$, Julia Budzyńska ${ }^{1, A-D \oplus}$, \\ Magdalena Woźniak ${ }^{1, E-F} \odot$ \\ ${ }^{1}$ Medical University, Lublin, Poland \\ A - Research concept and design, B - Collection and/or assembly of data, C - Data analysis and interpretation, \\ $D$ - Writing the article, E - Critical revision of the article, F-Final approval of the article
}

Karska K, Kozioł I, Leśniewska M, Budzyńska J, Woźniak M. Radiological features of perivascular epithelioid cell tumours (PEComas) in a paediatric patient - Case report. J Pre-Clin Clin Res. 2022; 16(1): 9-12. doi: 10.26444/jpccr/146536

\section{Abstract}

Introduction. Perivascular epithelioid cell tumour (PEComa) is a rare family of mesenchymal tumours composed of epithelioid cells. Due to its very rare occurrence, little information is available on the imaging characteristics of this type of lesion.

Case Report. A 16-year-old patient was admitted for diagnosis of an incidentally found lesion of unclear origin on MRI of the lumbar spine. Targeted MRI revealed a pathological solid-cystic mass within the pelis, in the left rectovesical pouch, infiltrating the bladder wall. A biopsy of the tumour was performed. On histopathological examination, a PEComa tumour was diagnosed. Control examinations were performed. The tumour was treated with embolization, surgical resection and Sirolimus therapy.

Conclusions. The tumour showed the characteristic features of PEC-oma on imaging studies reported in the literature. Radiological diagnosis is not fully specific, therefore histopathological examination is necessary for a definitive diagnosis.

\section{Key words}

MRI, CT, PEComa, perivascular tumour

\section{INTRODUCTION}

Perivascular epithelioid cell tumours (PEComas) are a very rare group of neoplasms, newly classified by the WHO in 2002, which are defined as mesenchymal tumours composed of perivascular epithelioid cells with characteristic histological and immunohistochemical features $[1,2]$. This group includes: angiomyolipoma of the kidneys, tumour of the lung, lymphangioleiomyomatosis, clear cell myomelanocytic tumour of the falciform ligament/ligamentum teres, and atypical clear cell tumours of the pancreas, rectum, peritoneum, uterus, vulva or heart $[3,4]$.

Liebow and Castleman first described PEComa as a benign lung tumour in 1971. Most of these tumours are thought to have benign behaviour [5]. To date, approximately 100 cases of PEComas have been described, mostly in the form of case reports. The most common location of PEComas was the genitourinary system [6]. Due to the rarity of these lesions there is little information about their characteristics, which, in turn, hinders their proper diagnosis. There is an insufficient number of reports characterizing this type of tumour on computed tomography (CT) or magnetic resonance imaging (MRI). Therefore, the knowledge about imaging PEComas and their radiological features needs further development [1].

Address for correspondence: Klaudia Karska, Medical University in Lublin, Pediatric Radiology Department, ul. Gębali 6, Lublin, Poland

E-mail: klaudiakarska@umlub.pl

Received: 06.10.2021; accepted: 08.02.2022; first published: 24.02 .2022

\section{CASE REPORT}

A 16-year-old male with lumbar spine pain underwent a review MRI examination. A lesion of unclear origin in the lesser pelvis was found. The patient was admitted to a Children's Hospital for further diagnostics (Figure 1).

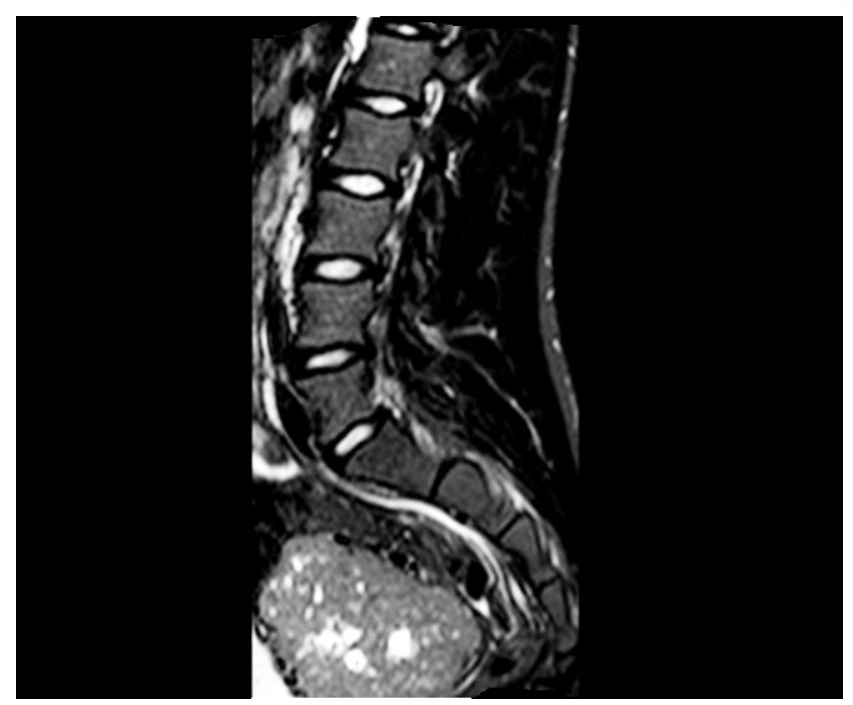

Figure 1. First MRI scan, $T 2 w$ in sagittal plane: pathological mass in the pelvis

Ultrasonography examination revealed an oval, pathological mass of intermediate heterogeneous echogenicity, measuring approximately $84 \times 61 \times 86 \mathrm{~mm}(\mathrm{AP} \times \mathrm{RL} \times \mathrm{CC})$, with a volume of approximately $229 \mathrm{ml}$. The lesion was located posterior to the bladder and caused pressure on this organ. Two anechoic, heterogeneous fluid spaces up to $12 \mathrm{~mm}$ 
in diameter were visible within the lesion. Colour Doppler examination showed a mild vascular flow within the lesion. No free fluid was detected in the abdominal cavity (Fig. 2).

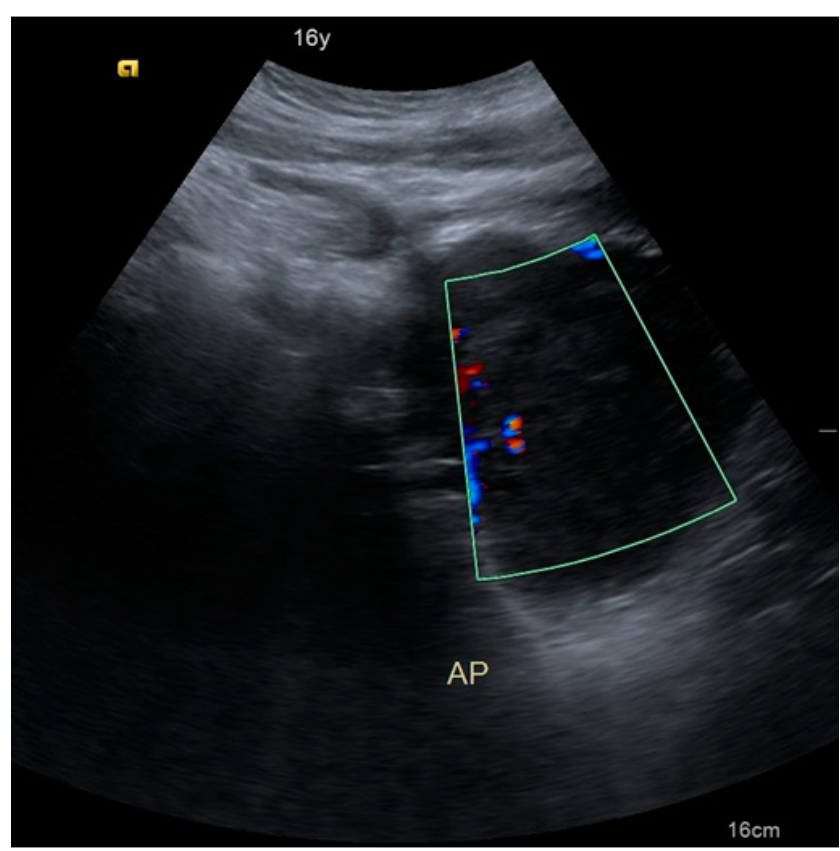

Figure 2. Ultrasound: heterogeneous fluid spaces and vessel segments in Colour Doppler

Pelvic MRI was performed with Siemens Magnetom Aera $481.5 \mathrm{~T}$ and Gadovist contrast agent (in amount of $7.5 \mathrm{ml}$ ) was used. A pathological mass was visualized in the left rectovesical pouch, measuring $98 \times 68 \times 78 \mathrm{~mm}(\mathrm{AP} \times \mathrm{RL} \times \mathrm{CC})$. The lesion infiltrated the bladder wall and caused shifting to the right side. Urethra and prostate were within normal limits. Multiple fluid spaces with fluid levels were seen within the lesion. In the periphery of the lesion, blood vessels pressed by the tumour were visualized. After the administration of a paramagnetic contrast agent, a non-intense, heterogeneous post-contrast enhancement of the lesion was observed. In DWI sequence, the lesion revealed restricted diffusion. A $6 \times 3 \mathrm{~mm}$ lymph node undergoing post-contrast enhancement was seen anteriorly to the described lesion. Some fluid space in the rectovesical pouch was also visualized (Fig. 3).
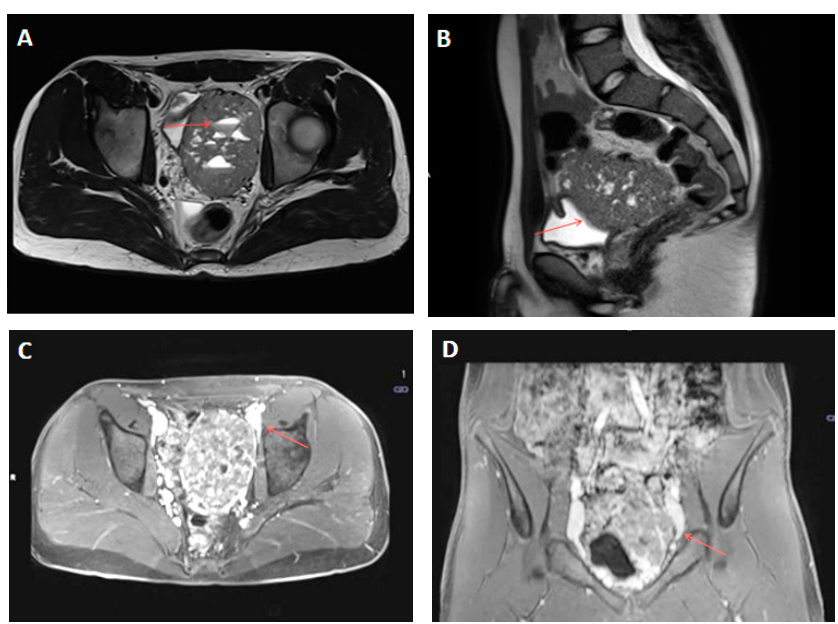

Figure 3. MRI: (A) T2w. Fluid levels inside of lesion. (B) T2w. Probable infiltration of the bladder wall. (C, D) Post-contrast T1w. Impression on blood vessels visible
Biopsy of the tumour was performed, and histopathological examination confirmed a Perivascular Epithelioid Cell Tumour, consisting of spindled and epithelioid cells with clear cytoplasm. In immunohistochemistry, positive staining was found using HMB45, SMA and INI1, positive/negative in SOX10, negative/positive in MelanA and negative in S100. Malignancy of tumour was not clearly determined in the given result.

After one month, abdominal and pelvic CT was performed with Siemens Definition AS+ before and after contrast agent administration. A $112 \times 65 \times 86 \mathrm{~mm}(\mathrm{AP} \times \mathrm{RL} \times \mathrm{CC})$ solidcystic tumour with a central necrotic zone was seen in the lesser pelvis. The tumour displaced the urinary bladder and infiltration of the bladder wall could not be excluded. The periphery of the lesion showed compressed blood vessels, as observed in MRI examination. The lesion underwent irregular post-contrast enhancement. A lymph node measuring $11 \times 9 \mathrm{~mm}$ was seen anteriorly to the described lesion, which also underwent post-contrast enhancement. Enlargement of the para-aortic lymph nodes up to $6 \mathrm{~mm}$ in the short axis was visualized. Chest CT with contrast showed two paratracheal lymph nodes (group 4R) measuring approximately $13 \times 6 \mathrm{~mm}$ and $8 \times 4 \mathrm{~mm}$ and axillary nodes up to $6-7 \mathrm{~mm}$ in the short axis (Fig. 4).
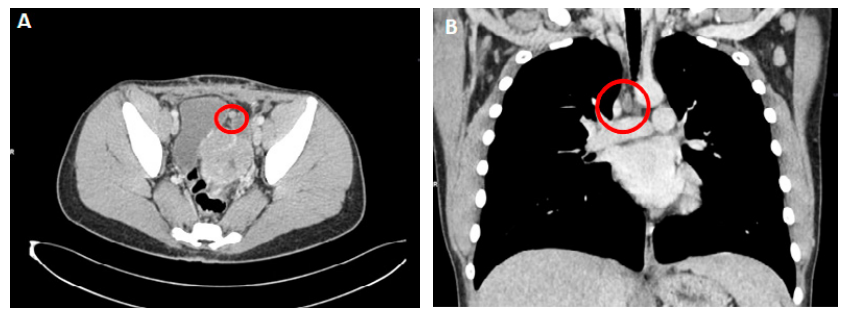

Figure 4. CT: (A) Enlarged lymph node with post-contrast enhancement. (B) Minor enlargement of distant lymph nodes.

After six months, pre-surgery embolization was performed as a means of decreasing the risk of haemorrhage during surgical removal of the tumour. After administration of a local anesthetic with lidocaine, catheterization with RUC (Roberts catheter) was performed using Seldinger's technique, and embolization material (Embozene Boston Scientific 700 and $900 \mu \mathrm{m}$ ) was administrated to the tumour vasculature. Control angiographic examination proved the technical success of the procedure.

After embolization, abdominal and pelvic CT with contrast medium was performed as a control examination. The non-contrast phase showed hyperdense areas along the course of the vessels - presence of the embolization agent. An irregular area in the centre of the lesion (probably embolization agent or bleeding area) and some gas bubbles were seen. The AP and CC dimensions of the lesion regressed slightly and the RL dimension progressed. The size of the tumour was approximately $99 \times 80 \times 82 \mathrm{~mm}(\mathrm{AP} \times \mathrm{RL} \times \mathrm{CC})$. The post-contrast enhancement of the lesion was less intensive in comparison to the previous studies, mostly in the perimeter. Post-contrast enhancing of a $5 \times 4 \mathrm{~mm}$ lymph node was visualized anteriorly to the described lesion (regression in size) (Fig. 5). Lymph nodes in the chest also regressed in size.

A week after embolization, subtotal removal of the tumour was performed via open surgery, sparing the bladder and leaving some residual mass in its proximity. 

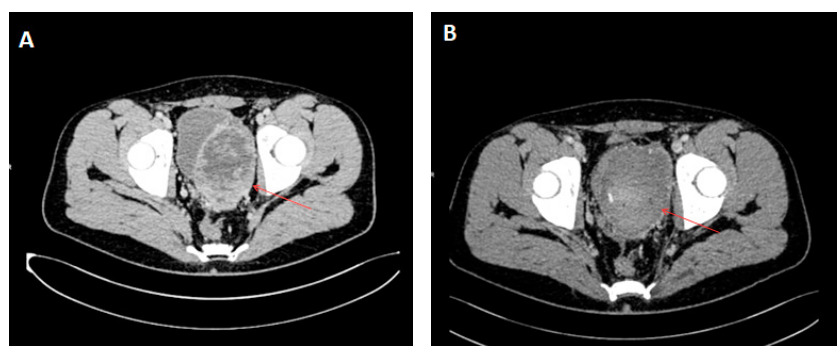

Figure 5. Post-contrast CT: (A) Before embolization. (B) After embolization

In post-surgery MR examination, regrowth of the tumour was observed, possibly due to surgery being only a subtotal resection. Lymph nodes in the chest continued to be monitored as their size had decreased in subsequent examinations.

After consultation with a national specialist of rare tumours, the patient remains under treatment with Sirolimus and the medicine blood load is being monitored.

Further control radiographic examinations are being scheduled.

\section{DISCUSSION}

As described previously, the age of patients diagnosed with PEComa tumours ranges from 14- 80 years. However, the peak incidence appears to be between the ages of 30-50. Tumours are more common in the females. The sizes of tumours reported in the literature range from $1.5-18 \mathrm{~cm}$, with the median size being between $4-5 \mathrm{~cm}[1,6,7]$.

Many locations of PEComa have been described including kidney, liver, lung, pancreas, palate, reproductive organs and soft tissues of the retroperitoneal space. The uterus is considered the most common location $[1,4,5]$. In the case described above, the tumour developed in a 16-year-old male patient, reaching $11.2 \mathrm{~cm}$ in size and being localized in the lesser pelvis in the rectovesical pouch.

Preoperative imaging with CT and MRI is not specific and is not sensitive enough to allow diagnosis of PEComa. It has been shown that the diagnosis was given in $31.3 \%$ of patients using CT, and $40 \%$ using MRI [1]. In case of PEComas, which occurred in the pelvic region, diagnostics were extended with ultrasound examination.

Using the ultrasound diagnostic tool, the tumours were visible as heterogeneous masses with solid and cystic components. Areas of necrosis have been observed in the larger tumours. The margins of the lesion were sharply demarcated in some cases and blurred in others. Colour Doppler studies have visualized mild vascular flow within the lesion $[2,6,8]$. A similar image was visualized by ultrasound in the case described.

Non-contrast MRI in patients with PEComa has shown hypointense masses in T1-weighted imaging and hyperintense masses in T2-weighted imaging $[1,4,8]$. PEComa tumours are usually described as solid masses with occasional cystic changes $[2,4,7]$; however, available studies suggest that PEComa can also take the form of a multicystic mass with fluid-fluid levels [6]. Most tumours were well demarcated and characterized by regular shape and heterogeneous contrast enhancement with late washout. MRI using a paramagnetic contrast agent has shown that most of the described masses are characterized by heterogeneous intensity and significant enhancement in the arterial and venous phases $[1,3]$. In the presented case, the lesion was well demarcated, regular in outline and solid-cystic in nature. It was characterized by heterogeneous post-contrast enhancement. Internal septa of varying thickness may be present within the tumour mass, as well as enhancing areas of necrosis in the centre of the lesion [7]. In some cases of PEComas, the presence of high intensity signal of fat in T1-weighted images has been described [6]. Moreover, the high signal intensity in the T1w sequence within the mass may be due to the presence of a haemorrhagic change or a protein-like fluid [2]. In the presented patient, only areas of necrosis in the centre of the lesion and presence of fluid spaces within the lesion were visualized.

On non-contrast CT, a regular shape and distinct borders with solid and cystic elements characterize PEComas. Necrosis may be present in large tumours. The vast majority are characterized by heterogeneous contrast agent uptake, intensified in solid elements $[1,4,6,8]$. The enhancement was significant in the arterial and venous phases, and slightly smaller in studies with delayed phase on CT imaging [1,7]. These features were also found in the CT scan performed in the presented patient. No enhancement was observed in the tumour where necrosis was predominant. Occasionally, there were tumours isodense to fat and with the presence of calcifications. In a few cases, vascular invasion was observed $[6,7]$, but these features were not observed in the presented case. Only two cases have been described with the occurrence of pelvic fluid [2]. In the patient described, a small amount of fluid was found in the rectovesical pouch.

Although most such tumours are benign and surgical resection is often sufficient for a complete cure, in a minority of cases, PEComas can show aggressive features: local recurrence, distant metastases - more often to the lungs, liver and peritoneum [4]. There are several reports of distant metastasis after surgical resection of primary tumours [1]. In the presented patient, local recurrence was observed after surgical resection. The classification criteria proposed by Folpe et al. assign these tumours to three categories of malignancy: benign, of uncertain malignant potential, and malignant. These criteria include tumour size $\geq 5 \mathrm{~cm}$, infiltrative growth pattern, high nuclear cellularity, mitotic index $>1 / 50$ high power fields (HPF), necrosis and vascular invasion. 'Benign' is defined as tumours without features typical of malignancy. Tumours are defined by the above criteria as 'malignant' if they clearly exhibit two or more of the atypical features listed above. However, meeting the above criteria is not synonymous with actual malignancy potential. Malignant PEComas are a highly aggressive disease with a high mortality rate $[8,9,10]$. The described PEComa occurring in the presented patient showed features of malignancy: size of the lesion $11 \mathrm{~cm}$, infiltration of the bladder wall and presence of necrosis. Whether these tumours can be considered benign or whether they all may carry some intrinsic risk of malignancy remains controversial [7]. Considering the above, long-term periodic follow-up is justified in all patients diagnosed with PEComa [1].

There are few descriptions of the radiological appearance of PEComa to identify specific diagnostic imaging features. Among the descriptions available in the literature, many tumours have a non-specific radiological character, which precludes the establishment of general criteria that would allow a definitive diagnosis. The heterogeneous appearance of PEComas is partly related to areas of cystic degeneration and/ 
or necrosis. This explains the heterogeneous enhancement in cross-sectional studies with rapid enhancement in the solid parts of tumours, and this is the description that has been used in many cases and large cohort stu, as well as in the presented case [2]. With more cases diagnosed in numerous new locations, the diagnostic criteria for PEComas are still evolving [5].

\section{CONCLUSIONS}

PEComas are a new group of mesenchymal tumours that may be considered in the differential diagnosis of solid and cystic masses in paediatric patients. The tumour presented above contained features of PEComa on imaging studies as described in the literature. The lesion was a solid-cystic, with a rather regular shape, distinct border, and the presence of necrosis and calcifications. These are not pathognomic characteristics reserved only for this type of tumour, therefore radiological diagnosis is not fully specific and histopathological examination is necessary for a definitive diagnosis

It is extremely important to document and evaluate PEComa cases, especially those with a longer clinical followup, to be able to better understand the biology of the lesion and to predict the possible future behaviour of these tumours.

\section{REFERENCES}

1. Tan Y, Zhang H, Xiao EH. Perivascular epithelioid cell tumour: dynamic CT, MRI and clinicopathological characteristics-analysis of 32 cases and review of the literature. Clin Radiol. 2013 Jun; 68(6): 555-61. doi: 10.1016/j.crad.2012.10.021. Epub 2012 Dec 11. PMID: 23245276.
2.Diestelkamp T, Mikes Z, Wilson-Smith R, Germaine P. Radiological findings of two neoplasms with perivascular epithelioid cell differentiation. Radiol Case Rep. 2017 Aug 19; 12(4): 845-849. doi: 10.1016/j.radcr.2017.06.001 PMID: 29484084; PMCID: PMC5823291.

3. Handa A, Fujita K, Kono T, Komori K, Hirobe S, Fukuzawa R. Radiological findings of perivascular epithelioid cell tumour (PEComa) of the falciform ligament. J Med Imaging Radiat Oncol. 2016 Dec; 60(6): 741-743. doi: 10.1111/1754-9485.12460 Epub 2016 Apr 20. PMID: 27094767.

4. Gkizas CV, Tsili AC, Katsios C, Argyropoulou MI. Perirenal PEComa: Computed Tomography Findings and Differential Diagnosis. J Clin Imaging Sci. 2015 Dec 31; 5: 69. doi: 10.4103/2156-7514.172977. PMID: 26900493; PMCID: PMC4736062.

5. Zhao J, Teng H, Zhao R, Ding W, Yu K, Zhu L, Zhang J, Han Y. Malignant perivascular epithelioid cell tumour of the lung synchronous with a primary adenocarcinoma: one case report and review of the literature. BMC Cancer. 2019 Mar 15; 19(1): 235. doi:10.1186/s12885-019-5383-0 PMID: 30876389; PMCID: PMC6419825.

6. Tirumani SH, Shinagare AB, Hargreaves J, Jagannathan JP, Hornick JL, Wagner AJ, Ramaiya NH. Imaging features of primary and metastatic malignant perivascular epithelioid cell tumours. AJR Am J Roentgenol. 2014 Feb; 202(2): 252-8. doi: 10.2214/AJR.13.10909 PMID: 24450662.

7.Xuesong D, Hong G, Weiguo Z. Bladder Perivascular Epithelioid Cell Tumour: Dynamic CT and MRI Presentation of 2 Cases With 2-year Follow-up and Review of the Literature. Clin Genitourin Cancer. 2019 Oct; 17(5): e916-e922. doi: 10.1016/j.clgc.2019.06.016 Epub 2019 Jul 2. PMID: 31327725.

8. Faria SC, Elsherif SB, Sagebiel T, Cox V, Rao B, Lall C, Bhosale PR. Ischiorectal fossa: benign and malignant neoplasms of this "ignored" radiological anatomical space. Abdom Radiol (NY). 2019 May; 44(5): 1644-1674. doi: 10.1007/s00261-019-01930-7 PMID: 30955068.

9. Musella A, De Felice F, Kyriacou AK, Barletta F, Di Matteo FM, Marchetti C, Izzo L, Monti M, Benedetti Panici P, Redler A, D’Andrea V. Perivascular epithelioid cell neoplasm (PEComa) of the uterus: A systematic review. Int J Surg. 2015 Jul; 19: 1-5. doi: 10.1016/j. ijsu.2015.05.002 Epub 2015 May 14. PMID: 25981307.

10. Nishio N, Kido A, Minamiguchi S, Kiguchi K, Kurata Y, Nakao KK, Kuwahara R, Yajima R, Otani S, Mandai M, Togashi K, Minami M. MR findings of uterine PEComa in patients with tuberous sclerosis: report of two cases. Abdom Radiol (NY). 2019 Apr; 44(4): 1256-1260. doi: 10.1007/s00261-019-01918-3 PMID: 30778737. 